\title{
How not to publish structural results
}

\author{
Alexander Wlodawer \\ Macromolecular Crystallography Laboratory, National Cancer Institute, Frederick, MD 21702, USA \\ wlodawer@nih.gov
}

High-resolution macromolecular structures determined using crystallography, NMR, and cryo-EM provide a gold standard for evaluation of important properties of biomolecules, but the quality of some structures, as well of their presentation, is not always fully acceptable. Whereas quality checking tools provided by the PDB during deposition process may flag some common problems, the resulting red flags are not always addressed by deposition authors. Some journals require that manuscripts be accompanied by validation reports in order to assist reviewers in evaluation of the validity of presented structures, whereas other journals do not have such requirements. Additionally, validation reports are more helpful in identifying global problems, while some local problems may not be apparent. Utilization of additional tools and interactive software might assist readers in making the best use of published structural data. Using examples extracted from the Protein Data Bank, as well as from journal publications, some common problems will be identified and suggestions will be made on how to avoid their reoccurrence.

Keywords: Protein Data Bank; structure quality; structure validation; journal policies 